\title{
Producción, uso y comercialización de especies aromáticas en la región Sumapaz, Cundinamarca
}

\section{Production, use and marketing of aromatic species in the Sumapaz region of Cundinamarca}

JOSÉ OMAR CARDONA ${ }^{1,3}$

JUAN CARLOS BARRIENTOS F.2

Compra y venta de plantas aromáticas, medicinales y condimentarías tradicionales en Fusagasugá, Cundinamarca.

Foto: J.0. Cardona

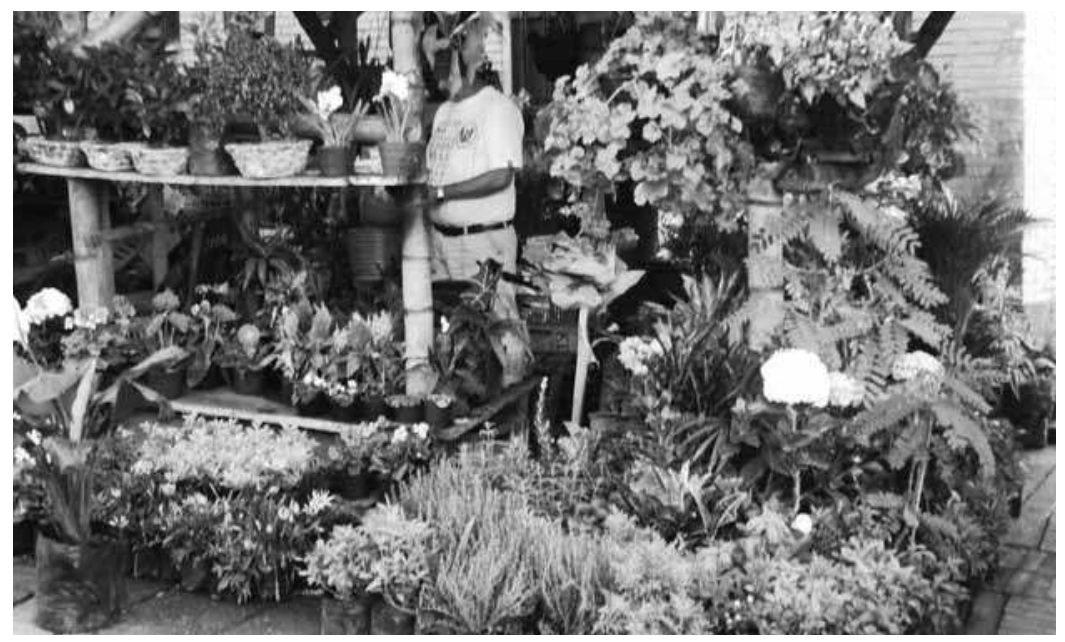

\section{RESUMEN}

En el marco del programa de investigación "Aprovechamiento de aceites esenciales de especies nativas y foráneas promisorias para uso agrícola en Colombia (2007V7163)" se realizó el presente trabajo guiado con el objetivo de: describir la producción, uso y comercialización de especies aromáticas en la región Sumapaz, Cundinamarca. La información que se requería provino de dos encuestas realizadas, una a 82 productores agrarios y otra a 19 comerciantes de aromáticas de la zona de estudio. Se identificaron 163 especies de plantas aromáticas, de las cuales más del 50\% de ellas son "no cultivadas". Las especies cultivadas, principalmente la yerbabuena, ruda, limonaria, cidrón, romero, toronjil, albahaca, manzanilla, tomillo y caléndula, son las más difundidas y comercializadas; aunque su producción es aún marginal. Las especies no cultivadas son recolectadas para su venta. El consumo y el uso tradicional de aromáticas se realizan principalmente en estado fresco y como medicamento respectivamente, pero también se ha encontrado usos como insecticida. La comercialización se realiza principalmente en plazas de mercado. El negocio es marginal por las altas tasas de pérdidas, los pequeños volúmenes de producción y comercialización, y el bajo o nulo valor agregado del producto.

Palabras clave adicionales: especie no cultivada,productores, recolectores, plaza de mercado, valor agregado.

Facultad de Ciencias Agropecuarias, Programa de Posgrado, Universidad Nacional de Colombia, Palmira (Colombia). Facultad de Agronomía, Departamento de Agronomía, Universidad Nacional de Colombia, Bogotá (Colombia).

3 Autor para correspondencia. jomarcardonam@unal.edu.co 


\section{ABSTRACT}

Under the research program "Development of Essential Oils from Promising Native and Foreign Species for Agricultural Use in Colombia (2007V7163)", this research was guided by the following objective: description of the production, use and marketing of aromatic species in the Sumapaz region of Cundinamarca. The required information came from two surveys, one conducted on 82 agricultural producers and the other on 19 aromatic merchants in the study area. We identified 163 species of herbs; of which it was approximated that over $50 \%$ are "uncultured". Cultivated species, mainly mint, rue, lemon grass, lemon verbena, rosemary, lemon balm, basil, chamomile, thyme and marigold, are the most widely used and marketed, although their production is still marginal. Uncultivated species are harvested for sale. Traditionally, aromatic herbs are usually consumed fresh and used as medicine, but people have also found uses as an insecticide. Marketing is done primarily in marketplaces. Business is marginal due to high loss rates, small volumes of production and marketing, and the low added value of the product.

Additional key words: uncultured species, producers, collectors, marketplace, added value.

Las plantas aromáticas se caracterizan por contener cantidades apreciables de compuestos químicos fácilmente perceptibles por el olfato (Bareño y Clavijo, 2006). Estos fitoquímicos, principalmente fenoles o sus derivados, se encuentran en toda la planta o en hojas, tallos, flores, frutos, raíces (Pacheco y Pohlan, 2006). Las plantas aromáticas son también llamadas "hierbas" aromáticas, dado su carácter herbáceo como la menta, el tomillo, el orégano; y en algunos casos plantas arbustivas como el romero, la ruda, entre otras; también las hay arbóreas como la canela, la nuez moscada y el eucalipto (Palacio, 2000). El Ministerio de Agricultura y Desarrollo Rural de Colombia (MADR), con la participación de productores, comerciantes, transformadores, consumidores e investigadores, conformó en 2004 la cadena de plantas aromáticas, medicinales, condimentarias y afines (PAMC), haciendo énfasis en 'usos' y potencial económico (Ministerio de Agricultura y Desarrollo Rural, 2009).
Las plantas aromáticas tienen usos condimentario, medicinal, ornamental, cosmético y otros. Las formas de uso/consumo son: en fresco, deshidratado y como aceite esencial (Portilla, 2007). El Centro de Investigación para el Desarrollo (CID) de la Universidad Nacional de Colombia, considera que los productos de las PAMC son utilizados: como plantas de consumo en fresco, para uso culinario y medicinal; como deshidratados o pulverizados, para la preparación de tisanas, uso condimentario y medicinal; como ingredientes naturales para la industria farmacéutica, cosmética y alimenticia; y como productos terminados con fines farmacéuticos, cosméticos y culinarios. El uso tradicional de las plantas aromáticas, un tanto diferente a su uso comercial, se basa en teorías, creencias y experiencias de los oriundos de las diferentes culturas, sean estas explicables o no. La ventaja de la tradición, en este caso el uso de las aromáticas, es que encierra un conocimiento empírico valioso que puede ser utilizado con fines comerciales; el riesgo está en que ese 
conocimiento, si no se formaliza o aprovecha, se puede perder (Ceuta, 2000; Aguilera, 2003; Arruda y Vieira, 2003; Marcus, 2003).

La información que se tiene sobre la producción colombiana de PAMC es referencial (Díaz, 2006). Por la cantidad de especies (> 200) bajo la denominación de plantas aromáticas es difícil determinar la producción y el consumo en Colombia. El MADR presenta datos relativos a especies cultivadas con fines comerciales (tabla 1). Las especies referenciadas son aquellas que actualmente se destinan en gran parte al mer- cado de exportación y una fracción al mercado nacional, entre ellas están: albahaca, tomillo, romero, cebollín, orégano, menta, estragón, mejorana, salvia, caléndula, manzanilla y hierbabuena (Bareño y Clavijo, 2006; Díaz, 2006 en: Ministerio de Agricultura y Desarrollo Rural; Acuerdo de competitividad, Cundinamarca, 2006; Repetto, 2005). La tabla 1 visualiza tanto la superficie de cultivo como la producción a nivel nacional mostrando, especialmente en los últimos años, una tendencia de notable incremento, seis veces y 25 veces respectivamente. Muestra también que el renglón pro-

Tabla 1. Área sembrada (ha) y producción en fresco (t) de plantas aromáticas, medicinales y condimentarias por departamento y año en Colombia (1997-2008).

\begin{tabular}{|c|c|c|c|c|c|c|c|c|c|c|c|}
\hline Departamento & Unidad & 1997 & 1998 & 1999 & 2000 & 2001 & 2002 & 2003 & 2004 & 2005 & $2008^{1}$ \\
\hline \multirow{2}{*}{ Antioquia } & ha & & & & & & & & & & 369 \\
\hline & $\mathrm{t}$ & & & & & & & & & & \\
\hline \multirow{2}{*}{ Boyacá } & ha & 5 & 15 & 15 & 15 & & & & & 18 & 30 \\
\hline & $\mathrm{t}$ & 31 & 90 & 90 & 90 & & & & & 10 & \\
\hline \multirow{2}{*}{ Cauca } & ha & & & 28 & 30 & & 60 & & & 160 & 97 \\
\hline & $\mathrm{t}$ & & & 28 & 30 & & 60 & & & 160 & \\
\hline \multirow[b]{2}{*}{ Cundinamarca } & ha & 142 & 140 & 170 & 190 & 280 & 289 & 316 & 331 & 354 & 454 \\
\hline & $\mathrm{t}$ & 828 & 913 & 1077 & 1113 & 3798 & 2217 & 2711 & 2294 & 1995 & \\
\hline \multirow{2}{*}{ Eje Cafetero } & ha & & & & & & & & & & 28 \\
\hline & $\mathrm{t}$ & & & & & & & & & & \\
\hline \multirow{2}{*}{$\begin{array}{l}\text { Norte } \\
\text { Santander }\end{array}$} & ha & 16 & 15 & 10 & 10 & 10 & 10 & 10 & 16 & 16 & 20 \\
\hline & $\mathrm{t}$ & 344 & 300 & 200 & 200 & 100 & 88 & 100 & 151 & 211 & \\
\hline \multirow{2}{*}{ Risaralda } & ha & 8 & 8 & 8 & 8 & 11 & 17 & 15 & 23 & & \\
\hline & $\mathrm{t}$ & 30 & 30 & 40 & 32 & 589 & 172 & 177 & 279 & & \\
\hline \multirow{2}{*}{ Tolima } & ha & & & & & & & & & & 48 \\
\hline & $\mathrm{t}$ & & & & & & & & & & \\
\hline \multirow{2}{*}{$\begin{array}{l}\text { Valle del } \\
\text { Cauca }\end{array}$} & ha & 23 & 107 & 116 & 120 & 103 & 116 & 120 & 114 & 165 & 200 \\
\hline & $\mathrm{t}$ & 70 & 274 & 294 & 300 & 535 & 619 & 674 & 1541 & 941 & \\
\hline \multirow{2}{*}{ Total Colombia } & ha & 194 & 285 & 347 & 358 & 404 & 492 & 461 & 484 & 713 & 1.246 \\
\hline & $\mathrm{t}$ & 1.303 & 1.607 & 1.729 & 1.675 & 5.022 & 3.156 & 3.662 & 4.265 & 3.237 & 33.127 \\
\hline \multicolumn{2}{|c|}{$\begin{array}{l}\text { Rendimiento promedio } \\
\left(\mathrm{t} \mathrm{ha} \mathrm{a}^{-1}\right)\end{array}$} & 6,700 & 5,600 & 5,000 & 4,700 & 12,400 & 6,400 & 7,900 & 8,800 & 4,500 & 26,600 \\
\hline
\end{tabular}

Fuente: Portilla, 2007; Ministerio de Agricultura y Desarrollo Rural, 2008. 
ductivo de aromáticas es relativamente nuevo, por lo que los volúmenes que se manejan son aún pequeños (Fernández, 2005; DNP, 2006; COBI/BIOTEC, 2007). Según reporte de Bareño y Clavijo (2006), los principales destinos de las aromáticas exportadas son: Estados Unidos de Norteamérica (74\%), Canadá (12\%), Inglaterra (8\%), Alemania (2\%), Holanda (2\%) y otros. Por otro lado, el mercado nacional, fuera de consumir las especies de exportación, también demanda: cúrcuma, limoncillo, toronjil, ruda, citronella, poleo y sábila. Los departamentos abastecedores del mercado nacional son: Cundinamarca, Antioquia, Valle del Cauca, Cauca y Tolima (Posso y Rodríguez, 2006; Rodríguez, 2004 en: MADR, 2008). La investigación realizada por Díaz (2003) sobre especies medicinales $y$ aromáticas ofrecidas en el mercado nacional indica que entre 50 y $60 \%$ corresponde a especies nativas, mientras que entre el 20 y $40 \%$ corresponden a especies naturalizadas. No se tiene información sobre porcentaje de especies nativas silvestres o cultivadas.

Rodríguez y Guzmán (2004) identificaron a tres tipos de oferentes primarios de hierbas aromáticas: productor convencional, productor orgánico y recolector de especies silvestres. Se le puede sumar un cuarto tipo, el productor recolector. De ahí es deducible que los productos vienen en diferentes calidades, y éstos se dirigen a los mercados correspondientes: (a) de exportación, (b) nacional producto fresco (restaurantes, supermercados, plazas de mercado de grandes ciudades, plazas de mercado de pequeñas ciudades) y (c) nacional industria. Una parte considerable de la producción comercial se destina al consumo en fresco. Esta forma de consumo tiene mucho que ver con la cultura gastronómica, principalmente de países importadores, con la mayor demanda. Las otras dos formas de consumo de aromáticas frescas es como medicamento en infusiones, baños, emplastos, y como materia prima o básica para laboratorios y tiendas naturistas que le dan al producto un valor agregado (Colombian National Press, 2006), transformán- dolas en productos medicinales o cosméticos (Fundacofan, 2004). Finalmente, vale la pena resaltar que hay especies aromáticas, nativas 0 introducidas, cultivadas o de recolección, cuyo uso, que se mantiene dentro una tradición regional, es poco conocido. A esto se debe sumar que el uso y aprovechamiento de las especies aromáticas en Colombia es aún relativamente reducido (García, 2005; Díaz, 2006).

La producción y recolección de hierbas aromáticas han sido y son en la región de Sumapaz parte importante de la tradición agrícola. De una manera general, la oferta de aromáticas en la región se caracteriza por bajo volumen y valor agregado al producto cosechado/recolectado, calidad no estandarizada y orientación al mercado local (Barrientos y Cardona, 2010). La provincia del Sumapaz tiene una gran diversidad de especies aromáticas entre cultivadas y silvestres y una tradición de cultivo y recolección de las mismas. Aunque los volúmenes actuales a nivel comercial en esta zona, para mercado nacional o exportación no son significativos, existe un gran potencial de oferta. Un objetivo de la cadena de PAMC, es obtener y promover en el futuro próximo productos elaborados o transformados como: principios activos y excipientes, fitoterapeuticos, cosméticos, aceites esenciales, biocidas y aromáticas y condimentarias (MADR, 2008). En concordancia, el proyecto de investigación "Conservación e identificación de especies aromáticas nativas promisorias por su contenido de aceites esenciales y con uso potencial para el sector agrícola colombiano" (Programa 2007V7163: "Aprovechamiento de aceites esenciales de especies nativas y foráneas promisorias para uso agrícola en Colombia", proyectos 2007V716350/51 presentados en la Cadena de Aromáticas del MADR/PTA, convocatorias I+D+I de 2006 y 2007 con vigencia 2008-2012) liderado por la Universidad Nacional de Colombia, sede Bogotá, se propone conocer las especies aromáticas presentes en la zona de estudio, sus características de producción, comercialización, uso y consumo. En ese sentido surgen algunas preguntas 
que sirven de guía para la presente investigación: ¿Cuáles y cuántas especies están presentes en la zona? ¿Cuáles especies se producen y cuáles se recolectan? ¿Cuáles son los usos/consumos más frecuentes de las especies aromáticas? ¿Cuáles especies y cómo se comercializan? El objetivo principal de la presente investigación fue determinar el uso tradicional y comercial de las especies aromáticas de la provincia Sumapaz, Cundinamarca. Como objetivos específicos se plantearon: a) determinar las especies de aromáticas presentes en la zona, b) diferenciar las especies por la forma de obtención y aprovechamiento, c) determinar el aprovechamiento comercial de las aromáticas, d) determinar el perfil de los productores y/o recolectores de aromáticas y e) describir el proceso de comercialización de aromáticas en la zona de estudio.

\section{METODOLOGÍA}

\section{Zona de estudio}

La provincia Sumapaz cuenta con 10 municipios: Fusagasugá (capital provincial), Silvania, Pasca, San Bernardo, Tibacuy, Venecia, Cabrera, Arbeláez y Pandi. Posee los tres pisos térmicos con temperaturas entre 2 y $24^{\circ} \mathrm{C}$; alturas entre 800 y $4.000 \mathrm{msnm}$, y precipitaciones de menos de 1.000 a más de $2.000 \mathrm{~mm}$ año ${ }^{-1}$; sus suelos son entre francos y arcillosos, de profundidad moderada y con buen contenido de materia orgánica, aptos para la agricultura (Incoder-Corpoica, 2005). Esta provincia se destaca además por: su ubicación geográfica estratégica (ecorregión central andina del país); su riqueza natural (Parque Natural del Sumapaz y Parque Natural Arqueológico de Quininí, Ecoparque Punto Verde y las Reservas Naturales de Silvania y del Sumapaz en Cabrera); su oferta ambiental (turismo-recreación-vivienda); su importancia vial (corredor vial centro-occidente del país); y por su desarrollo agropecuario intensivo con vocación hortofrutícola y ganadera (bovina, avícola, porcícola).

\section{Recolección de la información de campo}

Para el acopio de la información requerida se tomó como epicentro del estudio los 10 municipios de la provincia Sumapaz. Las fuentes de información sobre las especies presentes en toda la zona fueron los productores-colectores de aromáticas. Para lograr la información propuesta se aplicó una encuesta a través de un cuestionario semiestructurado de 30 preguntas divididas en cinco temas: a) información general sobre las plantas aromáticas presentes en la zona donde habita el encuestado, b) información sobre las plantas aromáticas que cultiva el agricultor, c) información sobre las plantas aromáticas que recolecta el agricultor, d) información general sobre el encuestado y e) información sobre la unidad productiva. Debido al desconocimiento el total de la población agraria de la zona de estudio y por la limitación de recursos se decidió tomar una muestra no probabilística consistente en por lo menos seis productores por municipio, $y$ en lo posible dos diferentes por vereda para tener información complementaria (tabla 2). Las encuestas se realizaron entre abril y de junio del 2008. Adicionalmente se realizó entrevistas a 19 comerciantes ubicados en las plazas de mercado

\begin{tabular}{|c|l|c|c|}
\hline Tabla 2. & \multicolumn{3}{|c|}{$\begin{array}{c}\text { Número de encuestados por vereda y } \\
\text { municipio. }\end{array}$} \\
\hline Nr. & Municipio & $\begin{array}{c}\text { Número de } \\
\text { veredas }\end{array}$ & $\begin{array}{c}\text { Número de } \\
\text { encuestados }\end{array}$ \\
\hline 1 & Arbeláez & 5 & 10 \\
\hline 2 & Cabrera & 3 & 6 \\
\hline 3 & Fusagasugá & 4 & 8 \\
\hline 4 & Granada & 3 & 6 \\
\hline 5 & Pandi & 5 & 8 \\
\hline 6 & Pasca & 6 & 10 \\
\hline 7 & San Bernardo & 4 & 8 \\
\hline 8 & Silvania & 4 & 8 \\
\hline 9 & Tibacuy & 4 & 8 \\
\hline 10 & Venecia & 6 & 10 \\
\hline Total & & 44 & 82 \\
\hline
\end{tabular}


de Fusagasugá (5), Pandi (3), Venecia (4), Pasca (2), Arbeláez (1) y San Bernardo (4) con el fin de determinar, a través de un cuestionario semiestructurado, las características del punto de venta, el origen y destino del producto vendido y la forma de uso de los consumidores. Esta encuesta se realizó entre noviembre y diciembre del 2008.

\section{Sistematización de la información de campo}

La información relacionada con la producción o recolección fue agrupada por especie y por encuestado. Las 82 encuestas permitieron crear una matriz de datos a partir de 163 especies (filas) y siete variables (columnas) de respuesta contenidas en seis grupos categorizantes (tabla 4). Para el caso de la encuesta de comercialización se elaboró una matriz de 76 filas (especies) y 30 columnas (variables descriptivas).

\section{Análisis de la información}

Se usaron FACTOR, PRINQUAL y \%plotit de SAS/STATv9 para crear biplots de preferencia de uso y conocimiento de las especies aromáticas locales. Se usó la estrategia Ward-MLM para agrupar especies, por uso y por habitante-usuario. Para categorizar especies tradicionales, se tomaron 21 componentes principales-CP- que explicaron el $100 \%$ de la respuesta obtenida en la prueba y se analizaron mediante la estrategia Ward-MLM. Con estos valores continuos (vectores propios) fue posible conformar grupos de especies y su importancia, evaluadas por aspectos socioculturales, socioeconómicos y de uso tradicional. De la matriz de comercialización se hizo un análisis de frecuencias de 30 variables.

\section{RESULTADOS Y DISCUSIÓN}

El uso tradicional de las plantas aromáticas se fundamenta en cultivar las especies más comerciales en pequeñas parcelas o jardines como algo marginal, y recolectar las especies no cultivadas y menos comerciales. Sus usos son principal- mente medicinales, pero también se destinan a su consumo como condimentos, usos esotéricos, infusiones e insecticidas. Poco más de la mitad de los encuestados comercializa especies aromáticas para, principalmente, consumo en fresco. Los volúmenes que se manejan son pequeños, la calidad no es su fuerte. Como agronegocio ocupa un lugar marginal dentro de la finca.

\section{Especies aromáticas presentes en la zona de estudio}

Se han encontrado 163 nombres comunes de especies consideradas aromáticas por los pobladores encuestados de la zona de estudio, de las cuales aproximadamente $73 \%$ ya tienen identificación taxonómica (tabla 3). No se descarta la posibilidad de encontrar entre el restante $27 \%$ de especies no identificadas taxonómicamente, aquellas que tengan más de un nombre común, lo que implicaría una disminución del total de especies encontradas.

Las 30 especies con mayor distribución geográfica en la zona de estudio son, en orden descendente: yerbabuena o hierbabuena (presente en 83\% de las unidades productivas de los encuestados), ruda, limonaria, cidrón, romero, toronjil, albahaca, manzanilla, tomillo, caléndula, matricaria, poleo, paico, sábila, ajenjo, altamisa, ortiga, perejil, apio, mejorana, orégano, verbena, sauco, hinojo, llantén, diente de león, barsalita, salvia, confrey y menta (presente en un $12 \%$ de las fincas). Su distribución parece estar directamente relacionada con su cultivo, su uso y valor comercial. Aproximadamente $52 \%$ de las especies resultantes de la encuesta fueron nombradas una sola vez (presente en 1,2\% de las unidades productivas de los encuestados), lo que refleja una muy poca distribución geográfica en la zona.

\section{Forma de obtención y uso}

De acuerdo con la tabla 4, hay 72 (43,94\%) especies que se cultivan, mientras que 91 especies $(56,06 \%)$ son "no cultivadas", tipo arvense que 


\section{Tabla 3. Especies de aromáticas encontradas en la zona.}

\begin{tabular}{|c|c|c|c|c|c|c|}
\hline Nombre local & Familia & Nombre científico & Usos & Menciones & Grupo & Frecuencia \\
\hline $\begin{array}{l}\text { Sígueme-Sígueme o } \\
\text { Querendona }\end{array}$ & & & Esotérico & 1 & 1 & 1,00 \\
\hline ViraVira & Asteraceae & Gnaphalium sp. & Medicinal & 5 & 1 & 4,00 \\
\hline Verdolaga & Portulacaceae & Portulaca oleracea & Medicinal & 6 & 1 & 4,00 \\
\hline Verbena & Verbenaceae & Verbena officinalis & Medicinal & 7 & 1 & 16,00 \\
\hline Tote & Cyperaceae & Cyperus strellata & Medicinal & 8 & 1 & 2,00 \\
\hline Toronjil & Lamiaceae & Melissa officinalis & Medicinal & 9 & 1 & 37,00 \\
\hline Pringamosa & Urticaceae & Urtica baccifera & Medicinal & 30 & 1 & 1,00 \\
\hline Poleo silvestre & Urticaceae & Urtica spp. & Medicinal & 31 & 1 & 1,00 \\
\hline Poleo canelo & Labaitae & Mentha pulegium & Medicinal/condimentario & 32 & 1 & 1,00 \\
\hline Poleo & Labaitae & Mentha spp. & Medicinal/condimentario & 33 & 1 & 26,00 \\
\hline Pino & Pinaceae & Pinus & Medicinal-industrial & 34 & 1 & 1,00 \\
\hline Pimentón & Solanaceae & Capsicum annuum & Medicinal/alimentario & 35 & 1 & 1,00 \\
\hline Perejil & Apiaceae & $\begin{array}{l}\text { Petroselinum } \\
\text { crispum }\end{array}$ & Medicinal & 37 & 1 & 18,00 \\
\hline Pepamora/yerbamora & Solanaceae & $\begin{array}{l}\text { Solanum } \\
\text { americanum }\end{array}$ & Medicinal & 38 & 1 & 1,00 \\
\hline Pepachina & & & & 39 & 1 & 1,00 \\
\hline Penca fique & Amaryllidaceae & Fourcraea spp. & Medicinal-industrial & 40 & 1 & 1,00 \\
\hline Pimpinela & & & & 41 & 1 & 1,00 \\
\hline Penicilina & & & & 42 & 1 & 2,00 \\
\hline Lima & & & & 78 & 1 & 1,00 \\
\hline Insulina & & $\begin{array}{l}\text { Boussingaultia } \\
\text { romosa }\end{array}$ & Medicinal & 84 & 1 & 4,00 \\
\hline Guayabo cimarrón & Myrtaceae & Psidium sp. & Alimentaria/medicinal & 88 & 1 & 1,00 \\
\hline Guaya Biche & Myrtaceae & Psidium guajava & Alimentaria/medicinal & 90 & 1 & 1,00 \\
\hline Guayaba & Myrtaceae & Psidium guajava & Alimentaria/medicinal & 91 & 1 & 1,00 \\
\hline Guasquín & & & & 93 & 1 & 1,00 \\
\hline Guasca & Labiatae & Salvia debilis & Condimentaria & 94 & 1 & 3,00 \\
\hline Guanábano & Annonaceae & Annona muricata & Alimentaria/medicinal & 95 & 1 & 1,00 \\
\hline Cicuta & Apiaceae & Conium maculatum & Medicinal & 122 & 1 & 8,00 \\
\hline Chitato & Malvaceae & Mutinga calabura & Medicinal & 124 & 1 & 1,00 \\
\hline Chipuca & & & & 126 & 1 & 1,00 \\
\hline Chipaca & Asteraceae & Bidens pilosa & Medicinal & 127 & 1 & 2,00 \\
\hline Bledo & Chenopodiaceae & Amaranthus sp. & Medicinal & 139 & 1 & 1,00 \\
\hline Apio & Apiaceae & Apium graveolens & Medicinal & 149 & 1 & 18,00 \\
\hline Anís & Apiaceae & Pimpinella anisum & Condimentaria & 150 & 1 & 4,00 \\
\hline Alfalfa & Fabaceae & Medicago sativa & Medicinal & 153 & 1 & 1,00 \\
\hline Albacastilla & & & & 155 & 1 & 1,00 \\
\hline Nombre local & Familia & Nombre científico & Usos & Menciones & Grupo & Frecuencia \\
\hline Tomillo & Lamiaceae & Thymus vulgaris & Condimentaria/medicinal & 10 & 2 & 30,00 \\
\hline Tinto & Solanaceae & Cestrum spp. & Medicinal & 11 & 2 & 1,00 \\
\hline $\begin{array}{l}\text { Tabaco de montaña o } \\
\text { de monte }\end{array}$ & Compositae & Árnica montana & Medicinal & 13 & 2 & 1,00 \\
\hline Sauce & Salicaceae & Salix spp. & Medicinal & 17 & 2 & 1,00 \\
\hline Salvia & Labiatae & Salvia officinalis & Medicinal & 21 & 2 & 1,00 \\
\hline Pronto alivio & Verbenaceae & Lippia alba & Medicinal & 29 & 2 & 4,00 \\
\hline
\end{tabular}


ESPECIES AROMÁTICAS EN LA REGIÓN SUMAPAZ, CUNDINAMARCA

\begin{tabular}{|c|c|c|c|c|c|c|}
\hline Perejil liso & Apiaceae & $\begin{array}{l}\text { Petroselinum } \\
\text { crispum }\end{array}$ & Medicinal & 36 & 2 & 1,00 \\
\hline Pmickay & Gramineae & Axonopus mikay & Medicinal & 43 & 2 & 1,00 \\
\hline Palitaria & Urticaceae & Parietaria debilis & Medicinal & 44 & 2 & 5,00 \\
\hline Palina & & & & 45 & 2 & 1,00 \\
\hline Pajarito & & & & 46 & 2 & 2,00 \\
\hline Paico & Amaranthaceae & $\begin{array}{l}\text { Chenopodium } \\
\text { ambrosoides }\end{array}$ & Medicinal/condimentario & 47 & 2 & 22,00 \\
\hline Ortiga negra & Urticaceae & Urtica spp. & Medicinal & 48 & 2 & 1,00 \\
\hline Ortiga espinosa & Urticaceae & Urtica spp. & Medicinal & 49 & 2 & 1,00 \\
\hline Mirto & Myrthaceae & Myrtus communis & Medicinal & 58 & 2 & 2,00 \\
\hline Guaba & Fabaceae & Inga edulis & Alimentaria/medicinal & 98 & 2 & 9,00 \\
\hline Frailejón & Asteraceae & Espeletia argéntea & Medicinal & 99 & 2 & 2,00 \\
\hline Flor de hamaca & & & & 100 & 2 & 1,00 \\
\hline Cerezo & Rosaceae & Prunus cerasus & Medicinal & 128 & 2 & 1,00 \\
\hline Cauto & & & & 129 & 2 & 1,00 \\
\hline Carare & & & & 130 & 2 & 1,00 \\
\hline Berenjena & Solanaceae & Solanum melongena & Medicinal & 141 & 2 & 1,00 \\
\hline Bejcolorado & Solanaceae & Solanum melongena & Medicinal & 142 & 2 & 1,00 \\
\hline Arnica & Asteraceae & Arnica spp. & Medicinal & 146 & 2 & 2,00 \\
\hline Ara negro & & & & 148 & 2 & 1,00 \\
\hline Alcachofa & Asteraceae & Cynara scolymus & Medicinal/condimentario & 154 & 2 & 2,00 \\
\hline Ajenjo & Asteraceae & Artemisia absinthium & Medicinal & 159 & 2 & 21,00 \\
\hline Aguacate & Lauraceae & Persea americana & Alimentaria/medicinal & 160 & 2 & 1,00 \\
\hline Abrecaminos & & & & 163 & 2 & 1,00 \\
\hline Nombre local & Familia & Nombre científico & Usos & Menciones & Grupo & Frecuencia \\
\hline Ruda & Rutaceae & Ruta graveolens & $\begin{array}{l}\text { Medicinal/esotérico/ } \\
\text { condimentario }\end{array}$ & 25 & 3 & 58,00 \\
\hline Ortiga blanca & Urticaceae & Urtica spp. & Medicinal & 50 & 3 & 1,00 \\
\hline Ortiga & Urticaceae & Urtica spp. & Medicinal & 51 & 3 & 21,00 \\
\hline Orégano & Lamiacae & Origanum vulgare & Medicinal/condimentario & 52 & 3 & 17,00 \\
\hline Naranjo & Rutaceae & Citrus $\times$ sinensis) & Alimentaria/medicinal & 54 & 3 & 1,00 \\
\hline Naranja agria & Rutaceae & Citrus $\times$ aurantium & Alimentaria/medicinal & 55 & 3 & 2,00 \\
\hline Muérdago & Lorantaceae & Viscum álbum & Medicinal & 56 & 3 & 1,00 \\
\hline Mochila rota & & & & 57 & 3 & 2,00 \\
\hline Menta negra & Lamiaceae & Mentha spp. & Medicinal/condimentario & 59 & 3 & 1,00 \\
\hline Menta & Lamiaceae & Mentha spp. & Medicinal/condimentario & 60 & 3 & 10,00 \\
\hline Mejorana & Lamiaceae & Thymus mastichina & Medicinal & 61 & 3 & 18,00 \\
\hline Gualandy & Leguminoseae & Jacaranda coerulea & Medicinal & 96 & 3 & 2,00 \\
\hline Guaco & Asteraceae & $\begin{array}{l}\text { Mikania laevigata } \\
\text { Sch.Bip.ex Baker }\end{array}$ & Medicinal & 97 & 3 & 1,00 \\
\hline Eucalipto & & & & 101 & 3 & 8,00 \\
\hline Espinaca & & & & 103 & 3 & 1,00 \\
\hline Drago & Agaváceas & Dracaena draco & Medicinal & 105 & 3 & 1,00 \\
\hline Diente de león & Asteraceae & Taraxacum officinale & Alimentaria/medicinal & 107 & 3 & 12,00 \\
\hline Destrancadera & & & & 108 & 3 & 6,00 \\
\hline Descanses & & & & 109 & 3 & 1,00 \\
\hline Colmonte & & & & 115 & 3 & 1,00 \\
\hline Chuchuhua & & & & 123 & 3 & 1,00 \\
\hline
\end{tabular}




\begin{tabular}{|c|c|c|c|c|c|c|}
\hline Chisaca & & & & 125 & 3 & 1,00 \\
\hline Caléndula & Asteraceae & Calendula officinalis & Medicinal & 131 & 3 & 29,00 \\
\hline Canelón & Myrsinaceae & Rapanea laetevirens & Medicinal & 132 & 3 & 4,00 \\
\hline Canelo & Winteraceae & Drimys winteri & Medicinal & 133 & 3 & 1,00 \\
\hline Berónica & & & & 140 & 3 & 1,00 \\
\hline Barsalita & & & & 143 & 3 & 11,00 \\
\hline Balso & Grossulariaceas & Ribes spp. & Medicinal & 145 & 3 & 1,00 \\
\hline Agrás & & & & 161 & 3 & 1,00 \\
\hline Nombre local & Familia & Nombre científico & Usos & Menciones & Grupo & Frecuencia \\
\hline Sauco & Adoxaceae & Sambucus & Medicinal & 16 & 4 & 16,00 \\
\hline Quina Roja & Amaranthaceae & $\begin{array}{l}\text { Chenopodium } \\
\text { quinoa }\end{array}$ & Alimentaria/medicinal & 28 & 4 & 1,00 \\
\hline Matricaria & Asteraceae & Matricaria recutita & Medicinal & 62 & 4 & 28,00 \\
\hline Mataratón & leguminoseae & Gliricidia sepium & Medicinal/insecticida & 63 & 4 & 2,00 \\
\hline Mastranto & Labiatae & Salvia scutellarioides & Medicinal & 64 & 4 & 3,00 \\
\hline Marrubio & lamiaceae & Marrubium vulgare & Medicinal & 65 & 4 & 1,00 \\
\hline Marihuana & Cannabinaceae & Cannabis sativa & Medicinal/psicoactiva & 66 & 4 & 1,00 \\
\hline Manzanilla & Asteraceae & $\begin{array}{l}\text { Chamaemelum } \\
\text { nobile }\end{array}$ & Medicinal & 67 & 4 & 34,00 \\
\hline Manoleon & & & & 68 & 4 & 1,00 \\
\hline Mango & Anacardiaceae & Mangifera & Alimentaria/medicinal & 69 & 4 & 2,00 \\
\hline Limonaria & Poaceae & $\begin{array}{l}\text { Cymbopogon } \\
\text { citratus }\end{array}$ & Medicinal/aromático & 75 & 4 & 55,00 \\
\hline Curalotodo & Primulaceae & Coris monspeliensis & Medicinal & 110 & 4 & 1,00 \\
\hline Guasimo & Moraceae & Guazuma ulmifolia & Medicinal & 111 & 4 & 1,00 \\
\hline Confrey & Boraginaceae & $\begin{array}{l}\text { Symphytum } \\
\text { officinale }\end{array}$ & Medicinal & 113 & 4 & 10,00 \\
\hline Cidra & Cucurbitaceae & Sechium edule & Alimentaria/medicinal & 121 & 4 & 1,00 \\
\hline Albahaca & Lamiaceae & Ocimum basilicum & Condimentaria & 156 & 4 & 35,00 \\
\hline Nombre local & Familia & Nombre científico & Usos & Menciones & Grupo & Frecuencia \\
\hline Yerbamora & Solanaceae & Solanum nigrum & Medicinal & 3 & 5 & 4,00 \\
\hline Té ibisco & Malvaceae & $\begin{array}{l}\text { Hibiscus rosa- } \\
\text { sinensis }\end{array}$ & Medicinal & 12 & 5 & 1,00 \\
\hline Suelda con Suelda & Rosaceae & Potentilla candicans & Medicinal & 14 & 5 & 7,00 \\
\hline Sorsilicio & & & & 15 & 5 & 1,00 \\
\hline Sanalotodo & Crasulaceae & Sedum telephium & Medicinal & 19 & 5 & 3,00 \\
\hline Salvia & Lamiaceae & Salvia officinalis & Medicinal & 20 & 5 & 11,00 \\
\hline Noni & Rubiaceae & Morinda citrifolia & Medicinal & 53 & 5 & 1,00 \\
\hline Malba & Malvaceae & malva negelcta & Medicinal & 70 & 5 & 5,00 \\
\hline Maguey & Agavaceae & Agave spp. & Medicinal & 71 & 5 & 1,00 \\
\hline Llantén & Plantaginaceae & Plantago lanceolata & Medicinal & 72 & 5 & 12,00 \\
\hline Llamarada & & & & 73 & 5 & 1,00 \\
\hline Linaza & Linaceae & Linum usitatissimum & Medicinal & 74 & 5 & 1,00 \\
\hline Limón & & Citrus $\times$ limón & Medicinal/condimentario & 77 & 5 & 4,00 \\
\hline Laurel & Lauraceae & Laurus nobilis & Medicinal & 80 & 5 & 8,00 \\
\hline Junquillo & & & & 81 & 5 & 1,00 \\
\hline Imperial & & & & 85 & 5 & 1,00 \\
\hline Guatila & Cucurbitaceae & Sechium edule & Alimentaria/medicinal & 92 & 5 & 1,00 \\
\hline Estevia & Asteraceae & stevia rebaudiana & Edulcolante & 102 & 5 & 1,00 \\
\hline
\end{tabular}




\begin{tabular}{|c|c|c|c|c|c|c|}
\hline Durazno & & & & 104 & 5 & 1,00 \\
\hline Dormidera & & & & 106 & 5 & 2,00 \\
\hline Cordoncillo & Piperaceae & Piper spp. & Medicinal & 112 & 5 & 6,00 \\
\hline Cola de caballo & Equisetaceae & Equisetum spp. & Medicinal & 114 & 5 & 7,00 \\
\hline Nombre local & Familia & Nombre cientifico & Usos & Menciones & Grupo & Frecuencia \\
\hline Cilantrón & & & & 116 & 5 & 4,00 \\
\hline CSanto & & & & 117 & 5 & 3,00 \\
\hline CMonte & & & & 118 & 5 & 1,00 \\
\hline Cajeto & Bignoniaceae & Delostoma tosea & Medicinal & 134 & 5 & 5,00 \\
\hline CadPerro & & & & 135 & 5 & 1,00 \\
\hline Brevo & & & & 136 & 5 & 1,00 \\
\hline Borraja & Boraginaceae & Borago officinalis & Medicinal & 137 & 5 & 5,00 \\
\hline Borrachero & Solanaceae & Datura stramonium & Medicinal & 138 & 5 & 2,00 \\
\hline Barbasco & Fabaceae & Lonchocarpus urucu & Medicinal/biocida & 144 & 5 & 3,00 \\
\hline Arcilla & & & & 147 & 5 & 1,00 \\
\hline Anamú & Fitolacaceae & Petiberia spp. & Medicinal & 151 & 5 & 3,00 \\
\hline Altamisa & Asteraceae & Achilea millefolium & Medicinal & 152 & 5 & 21,00 \\
\hline Ají & Solanaceae & Capsicum annuum & Alimentaria/medicinal & 158 & 5 & 2,00 \\
\hline Nombre local & Familia & Nombre científico & Usos & Menciones & Grupo & Frecuencia \\
\hline $\begin{array}{l}\text { Yerbabuena } 0 \\
\text { hierbabuena }\end{array}$ & Lamiaceae & Mentha spp. & Medicinal/condimentario & 2 & 6 & 68,00 \\
\hline Yerba del dolor & Hypericaceae & $\begin{array}{l}\text { Hypericum } \\
\text { perforatum }\end{array}$ & Medicinal & 4 & 6 & 8,00 \\
\hline Santa María & & & & 18 & 6 & 1,00 \\
\hline Salfante & & & & 22 & 6 & 1,00 \\
\hline Sábila & Asfodelaceae & Aloe barbadensis & Medicinal & 23 & 6 & 21,00 \\
\hline Ruibarbo & Polygonaceae & Rheum officinale & Medicinal & 24 & 6 & 1,00 \\
\hline Romero & lamiaceae & $\begin{array}{l}\text { Rosmarinus } \\
\text { officinale }\end{array}$ & Medicinal & 26 & 6 & 39,00 \\
\hline Riñonada & & & & 27 & 6 & 2,00 \\
\hline Limón Castilla & & & & 76 & 6 & 1,00 \\
\hline Lengua de vaca & Polygonaceae & Rumex sp. & Medicinal & 79 & 6 & 3,00 \\
\hline Jarilla negra & & & & 82 & 6 & 1,00 \\
\hline Jarilla blanca & & & & 83 & 6 & 1,00 \\
\hline Higuerón & Poligonaceae & Ruprechtia apétala & Medicinal & 86 & 6 & 1,00 \\
\hline Hinojo & Apiaceae & Foeniculum vulgare & Medicinal & 87 & 6 & 14,00 \\
\hline Guayabo & & Psidum guajava & Alimentaria/medicinal & 89 & 6 & 2,00 \\
\hline Cilantro & Apaceae & Coriandrum sativum & Condimentario/medicinal & 119 & 6 & 4,00 \\
\hline Cidrón & Lamiaceae & Aloysia citrodora & Medicinal & 120 & 6 & 49,00 \\
\hline Ajo & Alliaceae & Allium sativum & Medicinal/condimentario & 157 & 6 & 2,00 \\
\hline Abrojo & Asteraceae & Centaurea calcitrapa & Medicinal & 162 & 6 & 1,00 \\
\hline
\end{tabular}

son recolectadas para su uso/consumo y venta. La frecuencia de uso/consumo por los encuestados es relativamente baja (promedio $=7,28 \%$ ). Los grupos 4 y 6 tienen una frecuencia de uso media $(\sim 12,0 \%)$, el grupo 3 baja $(\sim 7,0 \%)$ y los grupos 1,2 y 5 muy baja ( 4,0\%). Aproximadamente el $83 \%$ de las especies se pueden obtener durante todo el año. Su uso es preferentemente como medicina $(75,67 \%)$, pero también se usa como condimentaria (4,63\%), como aromática $(2,22 \%)$, como insecticida $(0,95 \%)$, otros usos $(6,29 \%)$ y uso combinado (10,24\%). Con actividad insecticida se reportan verbena, sábila, ortiga, llantén, cola de caballo, cicuta, barbasco, altamisa y ají criollo. Cerca del $85 \%$ de las plantas aromáticas son de olor agradable o inoloras, lo que favorece su uso/consumo, que mayormente es en fresco. Por su concentración de aceites esenciales, 
Tabla 4. Variables categorizantes de PAMC en Sumapaz.

\begin{tabular}{|c|c|c|c|c|c|c|c|c|c|}
\hline \multicolumn{3}{|c|}{ Variable (Descripción) / Grupos } & 1 & 2 & 3 & 4 & 5 & 6 & Promedio \\
\hline \multirow{2}{*}{$\begin{array}{l}c 1 \\
c 2\end{array}$} & \multirow{2}{*}{$\begin{array}{l}\text { Frecuencia y } \\
\text { proporción de uso }\end{array}$} & Frecuencia de uso & 4,83 & 4,10 & 7,41 & 12,00 & 3,77 & 11,58 & 7,28 \\
\hline & & Porcentaje de uso & 0,46 & 0,39 & 0,71 & 1,15 & 0,36 & 1,11 & 0,70 \\
\hline c3 & \multirow{2}{*}{ Producción } & Cultivada & 42,46 & 43,30 & 41,46 & 41,67 & 45,03 & 49,71 & 43,94 \\
\hline c4 & & No cultivada & 57,54 & 56,70 & 58,54 & 58,33 & 54,97 & 50,29 & 56,06 \\
\hline c5 & \multirow{3}{*}{$\begin{array}{l}\text { Frecuencia de } \\
\text { recolección }\end{array}$} & Mensual & 12,35 & 10,05 & 15,83 & 20,77 & 12,06 & 19,70 & 15,13 \\
\hline c6 & & Anual & 1,06 & 0,61 & 1,71 & 6,95 & 1,21 & 1,42 & 2,16 \\
\hline c7 & & Todo el año & 86,59 & 89,33 & 82,45 & 72,28 & 86,73 & 78,88 & 82,71 \\
\hline c8 & \multirow{6}{*}{ Forma de uso } & Medicina & 69,80 & 85,67 & 76,82 & 62,15 & 78,66 & 80,92 & 75,67 \\
\hline c9 & & Insecticida & 0,89 & 0,00 & 0,16 & 0,00 & 4,39 & 0,25 & 0,95 \\
\hline c10 & & Condimentaria & 7,25 & 6,08 & 2,70 & 3,35 & 3,67 & 4,76 & 4,64 \\
\hline c11 & & Aromática & 0,71 & 0,00 & 2,45 & 8,11 & 1,09 & 0,97 & 2,22 \\
\hline c12 & & Otros & 2,86 & 1,72 & 5,96 & 9,38 & 7,71 & 10,10 & 6,29 \\
\hline c13 & & Combinada & 18,50 & 6,53 & 11,91 & 17,02 & 4,48 & 3,00 & 10,24 \\
\hline c14 & \multirow{5}{*}{ Aroma } & Agradable/D ${ }^{1}$ & 59,29 & 45,32 & 64,18 & 69,76 & 50,55 & 56,05 & 57,53 \\
\hline c15 & & Agradable/E² & 4,33 & 2,22 & 3,86 & 2,02 & 7,53 & 2,54 & 3,75 \\
\hline c16 & & Desagradable/D & 9,56 & 11,73 & 3,73 & 4,50 & 19,99 & 18,87 & 11,40 \\
\hline c17 & & Desagradable/E & 0,69 & 0,49 & 3,69 & 3,79 & 1,09 & 0,49 & 1,71 \\
\hline c18 & & Inolora & 26,12 & 40,23 & 24,54 & 19,92 & 20,83 & 22,04 & 25,61 \\
\hline c19 & \multirow{3}{*}{ Presentación } & Fresco & 93,39 & 83,51 & 91,31 & 75,98 & 94,56 & 93,54 & 88,72 \\
\hline $\mathrm{c} 20$ & & Deshidratado & 0,00 & 3,83 & 0,16 & 0,23 & 0,00 & 0,24 & 0,74 \\
\hline c21 & & Combinado & 6,61 & 12,66 & 8,53 & 23,79 & 5,44 & 6,22 & 10,54 \\
\hline c22 & \multirow{8}{*}{$\begin{array}{l}\text { Partes útiles de la } \\
\text { planta }\end{array}$} & Toda la planta & 15,80 & 16,67 & 12,21 & 3,74 & 14,89 & 2,79 & 11,02 \\
\hline c23 & & Hojas & 60,08 & 46,38 & 49,16 & 55,71 & 58,16 & 74,31 & 57,30 \\
\hline c24 & & Tallos & 0,67 & 0,00 & 0,28 & 0,34 & 0,71 & 0,08 & 0,35 \\
\hline c25 & & Raíz & 0,71 & 3,61 & 3,45 & 0,11 & 0,00 & 1,75 & 1,61 \\
\hline c26 & & Frutos & 2,86 & 8,62 & 3,45 & 12,50 & 2,14 & 0,00 & 4,93 \\
\hline c27 & & Flores & 0,00 & 1,72 & 0,71 & 2,90 & 4,00 & 2,77 & 2,02 \\
\hline c28 & & Semillas & 2,86 & 0,00 & 0,06 & 0,18 & 0,00 & 0,15 & 0,54 \\
\hline c29 & & Combinaciones & 17,02 & 23,00 & 30,69 & 24,52 & 20,09 & 18,16 & 22,25 \\
\hline
\end{tabular}

${ }^{1} \mathrm{D}=$ duradero; ${ }^{2} \mathrm{E}=$ efímero.

la parte más utilizada de estas aromáticas, en la mayoría de los casos, son las hojas. El aprovechamiento y uso de especies aromáticas en la zona de estudio es en su mayoría incipiente y marginal al no constituirse en su mayoría como cultivo principal ni alternativo. Esto está relacionado con aspectos técnicos y comerciales como son: el manejo empírico y, muchas veces, inadecuado de los cultivos; además, se refleja la baja demanda de estos productos (basados en su calidad, volumen y utilidad) en el mercado local y nacional.

\section{Uso tradicional y comercial de las aromáticas}

Todos los entrevistados utilizan o consumen de alguna manera las especies aromáticas. De las 82 fincas de la muestra, 47 (57\%) comercializan aromáticas, sea regular o irregularmente. Las fincas 
que venden volúmenes igual o menores a $5 \mathrm{~kg} /$ semana, que son la mayoría (70\%), ofrecen principalmente especies no cultivadas; mientras que las fincas que venden volúmenes iguales o mayores a $10 \mathrm{~kg} / \mathrm{semana}(21 \%)$, lo hacen con especies cultivadas (tabla 5). Las especies comerciales están reguladas por el mercado, el que se basa en los componentes de calidad, volumen y utilidad. Procesadores y exportadores no dan soporte técnico a pequeños productores, y las instituciones locales no tienen líneas ni programas de desarrollo tecnológico para los productores locales. La región Sumapaz presenta productores aislados (poca presencia de cooperación entre productores) con producciones aromáticas en parcelas pequeñas anexas a cultivos comerciales o jardines (caléndula, yerbabuena, manzanilla, limonaria y ruda), exhibiendo además otro grupo de aromáticas cuyo aprovechamiento es de tipo extractivo (destrancadera, diente-león, diosme, hinojo, mejorana, menta-local, verbena), presentándose como arvenses en toda la región, distribuidas por piso térmico. Respecto al uso tradicional, la mayoría de las especies tienen uso medicinal.

Se han determinado tres grupos de plantas: a) mayor uso tradicional y comercial como infusión o tisana: cidrón, limonaria y yerbabuena; b) uso medicinal, condimentario y esotérico: ruda, poleo, tomillo, toronjil, sábila; c) uso medicinal: destrancadera, diente de león, diosme, hinojo, mejorana, menta-local y verbena. El cidrón y la limonaria tienen características comerciales y de uso local, mientras que la yerbabuena sigue siendo de manejo y uso tradicional debido a su fácil cultivo y mantenimiento en jardines locales. El cidrón y la limonaria son menos frecuentes en jardines, pero tiene demanda comercial por procesadores y en plazas de mercado local. Por otra parte, la ruda tiene el mayor uso tradicional en fresco en plazas de mercado como aderezo, como brotes y flores frescas picadas; y esotérico para baños y riegos. Esta especie es la más cultivada en huertos caseros y la que más actividad comercial muestra en la región.

\section{Perfil de los productores o recolectores de aromáticas}

La región Sumapaz, al igual que muchas regiones cercanas a los (grandes) centros urbanos, está en una fase de transición de la actividad agraria, que tiene que ver con un menor uso de los recursos mano de obra, tierra y capital financiero en este sector. La agricultura ya no es la única o principal actividad económica de los pobladores. El tiempo de dedicación también se ha reducido notoriamente. A pesar de la aparente disponibilidad de mano de obra por el tamaño de las familias, esta se orienta a otros sectores más dinámicos. El género de los encuestados (mayormente mujeres) y la edad (la mayoría superan los 50 años), confirman esta tendencia. Pero las características que favorecen el aprovechamiento de las especies aromáticas son la edad, que puede implicar un amplio conocimiento empírico, y la experiencia de los encuestados en la zona y actividad agraria, pues la mayoría lleva más de 4 años en el lugar (tabla 6).

Con muy pocas excepciones, la mayoría de los encuestados reconocen especies aromáticas con uso medicinal en humanos y animales domésticos. Se identificaron tres grupos o clases de encuestados

Tabla 5. Oferta semanal de aromáticas en Sumapaz.

\begin{tabular}{|c|c|c|c|}
\hline \multirow{2}{*}{ Tipo de especie } & \multicolumn{3}{|c|}{ Oferta semanal por finca } \\
\hline & $0-5 \mathrm{~kg}$ & $5-10 \mathrm{~kg}$ & $>10 \mathrm{~kg}$ \\
\hline Cultivada & $55 \%$ & $13 \%$ & $32 \%$ \\
\hline No cultivada (extracción) & $81 \%$ & $6 \%$ & $13 \%$ \\
\hline Total ponderado & $70 \%$ & $9 \%$ & $21 \%$ \\
\hline
\end{tabular}


Tabla 6. Perfil socioeconómico de los productores/recolectores de aromáticas.

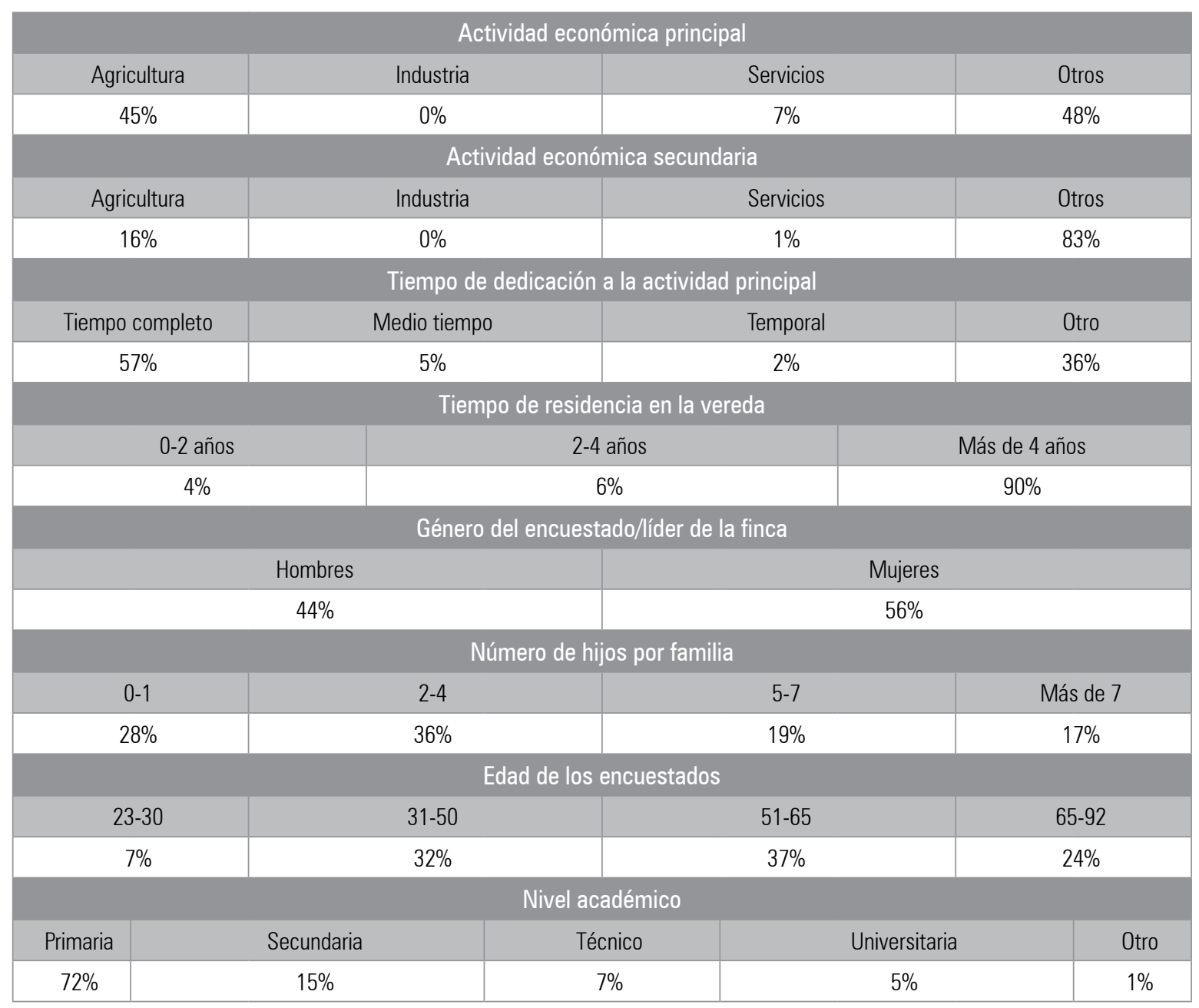

clasificados por su conocimiento, uso y aprovechamiento dado a las PAMC. El uso tradicional se da principalmente en adultos (30-60 años) y adultos mayores ( $>60$ años), la población joven (<30 años), aparentemente, está perdiendo tradición de uso de las especies presentes en su entorno, pues reporta menos especies y menos usos de las aromáticas. Hay especies con una clara preferencia de uso. Aunque la mayoría tienen uso medicinal, hay desconocimiento del verdadero valor etnobotánico, agroindustrial y comercial entre la población encuestada. Hay especies con uso muy exclusivo del cultivador/recolector y casi desconocido para el grupo encuestador y son usadas por un número reducido de personas. En general, el conocimiento y uso de las aromáticas es disper- so y ambiguo y una o más especies tienen nombre común diferente y uso distinto por subzona.

\section{Comercialización de aromáticas de la región Sumapaz}

La venta de aromáticas se realiza en un $80 \%$ en las plazas de mercado de los municipios de la zona de estudio, el 80\% del volumen de venta se concentra en la plaza de mercado de Fusagasugá y el restante $20 \%$ se distribuye entre Pandi, Venecia, Pasca, Arbeláez y San Bernardo. Las aromáticas son productos de venta marginal, pues no son lo más importante de los negocios donde estos suelen comercializarse. El 70\% se ofrecen con otros productos: $55 \%$ junto con frutas y ver- 
duras, $18 \%$ con especias, $14 \%$ con esotéricos y $13 \%$ con otros productos. E1 $80 \%$ de estos negocios funcionan todos los días, mientras que el $20 \%$ lo hacen solo los fines de semana o días de mercado. Los vendedores de especies aromáticas son mujeres en $55 \%$, $80 \%$ están entre 29 y 42 años, $10 \%$ son mayores a 50 años, $80 \%$ tienen solo educación primaria, el $20 \%$ tienen entre 2 y 5 años de experiencia y $40 \%$ más de cinco años. Un $50 \%$ de los vendedores solo vende, un $40 \%$ acopia y vende, un $3 \%$ clasifica y vende y un $8 \%$ acopia, procesa y vende. El 40\% de los comercializadores compran directamente del productor, $47 \%$ de los acopiadores y $12 \%$ de los acopiadoresproductores. El 95\% del volumen de aromáticas lo venden directamente a los consumidores y solo el $5 \%$ a los mayoristas o acopiadores. La tasa de venta está alrededor del $50 \%$, por lo que las pérdidas por no venta son altas. Los precios de compra-venta son bajos y sus fluctuaciones elevadas. Los consumidores son de estratos 1 , 2 y 3 . Los usos son $45 \%$ medicinales, $30 \%$ medicinal/condimentario y $12 \%$ medicinal/condimentario/insecticida. La especies más vendidas son, en forma descendente: yerbabuena, manzanilla, caléndula, limonaria, albahaca, cidrón, ruda, toronjil, poleo, tomillo, laurel, destrancadera, diente de león, diosme, hinojo, mejorana, paico, verbena, apio, menta y perejil.

\section{CONCLUSIONES Y RECOMENDACIONES}

La provincia Sumapaz es rica en especies aromáticas, tanto cultivadas como "no cultivadas". Tiene un alto potencial como fuente/reserva fitogenética.

La mayoría de las especies aromáticas son "no cultivadas". Su obtención para su uso, consumo o comercialización es a través de recolección.

Las 10 especies más difundidas en la zona de estudio son cultivadas. Su valor e importancia comercial incentiva su difusión. Más del 50\% de las especies no tiene actualmente relevancia co- mercial y su presencia en la zona es mucho menor que las anteriormente mencionadas.

Si bien los encuestados del Sumapaz conocen muchas especies aromáticas, su demanda para uso, consumo o comercialización es reducida. Las aromáticas se utilizan mayormente como medicamento y en menor proporción como condimento, aromática (infusión) e insecticida. Su mayor aprovechamiento radica en su venta como producto fresco ya que su transformación esta casi ausente. Las creencias y tradiciones conforman grupos de plantas alrededor de su uso medicinal $y$ alimentario. El conocimiento medicinal es empírico y con heterogeneidad en criterios de uso.

La comercialización de aromáticas, así como su cultivo, es marginal, es decir, en la mayoría de los casos no se constituye en cultivo y/o producto principal. Como el mercado local, hacia donde más se dirige, demanda volúmenes bajos, su oferta es de alrededor de $5 \mathrm{~kg} / \mathrm{semana}$. Además, solo poco menos de la mitad de los encuestados vende aromáticas.

La zona del Sumapaz está experimentando también un cambio en su sector agrario. La agricultura está dejando de ser la única o principal actividad económica. Como resultado de la migración campo/ciudad y violencia armada muchas fincas han quedado bajo la responsabilidad de mujeres y adultos mayores. Es probable que este fenómeno, como tendencia, reduzca el conocimiento y uso empírico de las aromáticas con el transcurrir del tiempo.

La comercialización de aromáticas se realiza principalmente en las plazas de mercado, siendo Fusagasugá la más concurrida. Los volúmenes que se manejan en los puestos de venta son pequeños, los precios fluctuantes y las pérdidas por no venta se ubican alrededor de $50 \%$.

El valor agregado de las aromáticas es bajo. Lo que más se realiza es la clasificación y en algunos casos la deshidratación. 


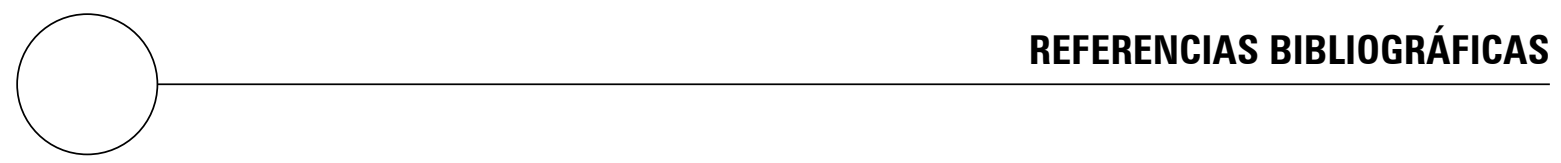

Aguilera, M. 2003. Las propiedades de las plantas. Últimas Noticias de Naturaleza y Salud, Montevideo.

Arruda, A. y F. Vieira. 2003. Ervanário Sao Francisco de Assis: Preparaçoes caseiras de remédios de plantas medicinais. Rede de Intercambio de Tecnologías Alternativas, Belo Horizonte, Brasil.

Bareño R.P. y P.J. Clavijo P. J. 2006. Hierbas aromáticas culinarias para exportación en fresco. En: Últimas tendencias en hierbas aromáticas para exportación en fresco, curso de extensión. Proyecto Hierbas Aromáticas. Facultad de Agronomía, Universidad Nacional de Colombia, Bogotá.

Barrientos F., J.C. y J.O. Cardona. 2010. Los pequeños productores están limitados para adoptar nuevos cultivos. Caso: hierbas aromáticas en la región Sumapaz, Cundinamarca. Agron. Colomb. 23(1), 99107.

Centro de Investigación para el Desarrollo (CID). 2008. Caracterización y evaluación de la cadena de plantas aromáticas, medicinales, condimentarias, aceites esenciales y afines en Colombia. Universidad Nacional de Colombia, Bogotá.

CEUTA, Red de plantas medicinales del Cono Sur. 2000. Uso popular de plantas medicinales en Pedernal: Investigación etnofarmacológica comunitaria con Grupo Pedernal de la Cooperativa Calmañana, Uruguay 1999-2000. CEUTA, Montevideo.

COBI/BIOTEC. 2007. Plan de la estrategia biorregión Valle del Cauca 2019. Puesta en marcha de la estrategia de biorregión Valle del Cauca Fase I. Consejo de Biorregión (COBI) Secretaria Técnica de Biorregión (Corp. BIOTEC) Centro de Investigaciones en Economía y Competitividad Internacional (CIECI). Pontificia Universidad Javeriana, Cali, Colombia.

Colombian National Press. 2006. Crece la demanda por productos con sello natural, a nivel mundial. Portafolio Comercio Exterior, Bogotá. Noviembre de 2006.

DNP. 2006. Agenda interna para la productividad y competitividad. Documento regional Valle del Cauca. Versión preliminar. Departamento Nacional de Planeación,Bogotá

Díaz J.A. 2006. Estrategia para tres sectores de biocomercio con estudios de mercado específicos. CAF. IAvH. Volumen VII. Bogotá.
Díaz J.A. 2003. Informe técnico. Caracterización del mercado colombiano de plantas medicinales y aromáticas. Instituto Alexander von Humboldt - Ministerio de Ambiente, Vivienda y Desarrollo Territorial, Bogotá.

Fernández, J. A. 2005. Programa jóvenes con empresa. Banco de Oportunidades de Negocio Valle del Cauca, Cali.

Fundacofan. 2004. Estudio internacional de mercado de plantas medicinales, aromáticas, condimentarias y sus subproductos. Informe final. Fundación Colombiana para la Farmacia Natural, Cali, Colombia.

García, P.L.F. 2005. Estudio de factibilidad para la producción de albahaca en el corregimiento de Pasuncha, municipio de Pacho, Cundinamarca. Trabajo de grado. Facultad de Ciencias Empresariales, Universidad de San Buenaventura, Bogotá.

Incoder-Corpoica. 2005. Zonificación agroecológica, evaluación económica y organización empresarial de sistemas de producción prioritarios en el área de desarrollo rural provincia del Sumapaz. Instituto Colombiano de Desarrollo Rural y Corpoica, Bogotá.

Marcus, A. 2004. De vicios y placeres. Ediciones de La Bruja, Zapala, Argentina.

Marcus, A. 2003. De malezas y buenezas. Ediciones de La Bruja, Zapala, Argentina.

Ministerio de Agricultura y Desarrollo Rural. 2009. Cadena de plantas aromáticas. En: http://www. minagricultura.gov.co/08cifras/08_Misi_Cadenas. aspx; consulta: julio de 2009.

Ministerio de Agricultura y Desarrollo Rural. 2008. Definición de la agenda prospectiva de investigación para la cadena productiva de plantas aromáticas, medicinales y condimentarias y afines con énfasis en ingredientes naturales para la industria cosmética en Colombia. Informe final. Bogotá.

Pacheco, B.A. y A.J. Pohlan. 2006. Efectos de plantas aromáticas sobre la estimulación de crecimiento y rendimiento en cultivos tropicales. En: Últimas tendencias en hierbas aromáticas para exportación en fresco, curso de extensión. Proyecto Hierbas Aromáticas. Facultad de Agronomía, Universidad Nacional de Colombia, Bogotá.

Palacio, N.L. 2000. Las plantas medicinales y aromáticas. Una alternativa de futuro para el desarrollo rural. 
Boletín Económico de ICE No. 2652, 1 al 4 de mayo de 2000.

Portilla, V.A. 2007. Entorno de la cadena productiva de las plantas aromáticas, medicinales y condimentarias en Colombia. En: Perspectivas del agronegocio de hierbas aromáticas, culinarias y medicinales. Proyecto Hierbas Aromáticas. Facultad de Agronomía, Universidad Nacional de Colombia, Bogotá.

Posso, P.S. y H.J. Rodríguez. 2006. Situación actual, agrícola e industrial del sector de plantas medicinales, aromáticas, condimentarias y afines en el Valle del Cauca. Documento convenio regional de competitividad de la cadena PMA y afines. Cali, Colombia.
Repetto, H. 2005. La cadena productiva de las plantas aromáticas, medicinales y condimentarías (PAM) en Cundinamarca. Asociación Colombiana para el Avance de la Ciencia (ACAC), Bogotá.

Rodríguez, H.J. y S.P. Guzmán. 2004. Identificación y caracterización de la oferta disponible de plantas medicinales y aromáticas en las iniciativas empresariales, comunidades organizadas y asociaciones más representativas que producen, acopian y/o transforman y comercializan en 9 departamentos. Fundacofan, Instituto Alexander von Humboldt, Ministerio de Ambiente, Vivienda y Desarrollo Territorial, Bogotá. 